\title{
The long-term impact on offspring of exposure to hyperglycaemia in utero due to maternal glucokinase gene mutations
}

\author{
R. Singh • E. R. Pearson • P. M. Clark • A. T. Hattersley
}

Received: 31 August 2006 / Accepted: 19 October 2006/ Published online: 10 January 2007

(C) Springer-Verlag 2007

\begin{abstract}
Aims/hypothesis There is strong evidence that maternal diabetes while offspring are in utero results in offspring beta cell dysfunction and diabetes or glucose intolerance. Offspring born to mothers with a mutation in the glucokinase gene $(G C K)$ are a good model for studying exposure to moderate hyperglycaemia, as mutation carriers have fasting hyperglycaemia throughout life including during pregnancy. We assessed the long term effects of exposure to maternal hyperglycaemia in utero on beta cell function and glucose tolerance in adult offspring.

Materials and methods We studied 86 adult offspring (mean age 40 years), 49 born to glucokinase mothers (exposed to hyperglycaemia in utero) and 37 born to glucokinase fathers (controls). We measured glucose tolerance during an OGTT and beta cell function using early insulin response (EIR); we also measured anthropometric data including birthweight.

Results Offspring of glucokinase mothers had a higher birthweight by $450 \mathrm{~g}(p<0.001)$, but no evidence of
\end{abstract}

\footnotetext{
R. Singh • E. R. Pearson · A. T. Hattersley $(\bowtie)$

Department of Molecular Medicine,

Institute of Biomedical and Clinical Sciences,

Peninsula Medical School,

Exeter EX2 5DW, UK

e-mail: Andrew.Hattersley@pms.ac.uk

\section{E. R. Pearson}

Division of Medicine and Therapeutics,

Ninewells Hospital \& Medical School,

Dundee, UK

P. M. Clark

The Regional Endocrine Laboratory,

University Hospital Birmingham NHS Foundation Trust,

Birmingham, UK
}

deterioration in glucose tolerance (2-h glucose 9.1 vs $8.6 \mathrm{mmol} / 1 \quad p=0.50$ ) or reduced beta cell function (log EIR 1.40 vs $1.26, p=0.11$ ) compared with offspring born to glucokinase fathers.

Conclusions/interpretation The marked increase in birthweight shows that offspring born to affected mothers were exposed to increased glycaemia in utero. Despite this, there was no evidence of altered beta cell function or glucose tolerance. As previous human examples of marked programming by hyperglycaemia in utero have been in genetically predisposed offspring, we propose that our finding reflects the lack of genetic predisposition in the offspring to progressive beta cell dysfunction.

Keywords Beta cell function - Diabetes $\cdot$ Fetal exposure . Glucokinase · Hyperglycaemia in utero · Maternal diabetes · Programming

\section{Abbreviation}

EIR early insulin response

\section{Introduction}

Type 2 diabetes is a complex disease with both environmental and genetic components. The role of the early intrauterine environment is controversial, but there is strong evidence that exposure to maternal diabetes in utero results in beta cell dysfunction and hyperglycaemia. Offspring born to Pima Indian mothers who were diabetic before pregnancy showed a very high prevalence $(45 \%)$ of diabetes before the age of 25 years when compared with those whose mothers developed diabetes after pregnancy (9\%) [1]. Diabetes in offspring exposed to diabetes in utero 
results predominantly from beta cell dysfunction [2]. Carriers of mutations in the MODY gene hepatocyte nuclear factor 1 alpha $(H N F 1 A)$ whose mothers had diabetes when they were in utero were diagnosed with diabetes 8 years earlier than those who inherited the mutation from their father [3]. Beta cell function was moderately reduced in white offspring born to non-diabetic mothers who developed type 2 diabetes before 50 years of age, compared with offspring of affected fathers [4]. Finally, a small study on offspring of type 1 diabetic parents showed increased glucose intolerance and beta cell dysfunction in offspring born to affected mothers compared with offspring of affected fathers [5].

Subjects with heterozygous mutations in the glucokinase gene $(G C K)$ have life-long, mild fasting hyperglycaemia (usually $5.5-8 \mathrm{mmol} / \mathrm{l}$ ) without associated insulin resistance or dyslipidaemia [6]. Offspring of mothers with glucokinase mutations are therefore exposed to consistent, moderate hyperglycaemia in utero, which differs from type 1 or type 2 diabetes in as far as the glucose levels are less variable and little altered by treatment [6]. Previous studies have shown a clear increase in birthweight associated with mothers having a glucokinase mutation [7], supporting the idea that offspring are exposed to moderate maternal hyperglycaemia in utero. Offspring of mothers with glucokinase mutations are therefore a good model for studying the impact of exposure to gestational hyperglycaemia. We compared beta cell function and glycaemia in offspring born to mothers with glucokinase mutations (exposed) with those of offspring whose fathers had glucokinase mutations (unexposed).

\section{Subjects and methods}

Subjects for clinical studies We recruited 83 offspring born to parents with glucokinase mutations from UK families. All offspring were over 16 years old and had only one parent with glucokinase mutation and no history of type 2 diabetes in first-degree relatives. Pregnant women and persons taking steroids were excluded. The study protocol was approved by the local research ethics committees, and the studies were performed in accordance with the Declaration of Helsinki. All participants gave written informed consent.

Methods for clinical studies Subjects fasted from $22.00 \mathrm{~h}$ the night before assessment, and avoided excessive exercise and alcohol for the previous $24 \mathrm{~h}$. Three plasma samples for insulin and glucose were collected from the fasting subjects before an OGTT. Additional plasma samples $(n=4)$ were taken at 30-min intervals following a 75-g oral glucose load. Body composition was assessed by bioelectrical impedance (Bodystat 1500; Bodystat, Douglas, Isle of Man, British Isles). Blood pressure and waist and hip measurements were taken. Details of birthweight and gestation were obtained from clinical notes or by maternal recall.

The primary endpoint was early insulin response (EIR) following OGTT, calculated using the formula (30-min insulin-mean fasting insulin)/(30-min glucose) [8]. Secondary endpoints were glucose tolerance during OGTT, $\mathrm{HbA}_{1 \mathrm{c}}$, and insulin sensitivity determined by the homeostasis model assessment computer model v2.2 [9].

Sample analysis Serum and plasma were stored at $-80^{\circ} \mathrm{C}$. All biochemical analyses were performed in the same UK laboratories, and insulin was measured by an immunoenzymometric assay (Insulin EASIA; Biosource-Invitrogen, Boston, MA, USA) calibrated against the International Reference Preparation 66/304 (http://www.nibsc.ac.uk), with no detectable cross-reactivity with intact proinsulin and $32-33$ split proinsulin. Plasma glucose was analysed by glucose oxidase methods and $\mathrm{HbA}_{1 \mathrm{c}}$ by using an in-house high-performance liquid chromatography method (normal range $4.0-5.5 \%$ ).

All study subjects were sequenced for glucokinase mutations. Proband genomic DNA samples were amplified using published primers for all exons and intron-exon boundaries of the glucokinase gene, while only the relevant primers for index mutations were used for all other familial DNA samples. PCR products were purified and sequenced using BigDye Terminator chemistry (Applied Biosystems, Warrington, UK). Reactions were analysed on a DNA sequencer (ABI Prism 3100; Applied Biosystems) and sequences compared using a mutation surveyor software (Mutation Surveyor v2.51; SoftGenetics, Pennsylvania, PA, USA).

Statistical analysis Offspring born to mothers with glucokinase mutations were compared with offspring born to fathers with glucokinase mutations. Distribution of sex, glucose intolerance and diabetic status among the two groups were compared using chi-squared tests. Data are presented as means with SEM. Data showing a skewed distribution were log-transformed. Mean age was compared using the Student's $t$-test. All other means were compared by analysis of covariance using age, sex, and mutation status as covariates. All tests were two-tailed and the significance level was 0.05 .

We calculated that to have $80 \%$ power to see the same deterioration in 2-h glucose $(1.5 \mathrm{mmol} / \mathrm{l})$ and EIR $(31 \%)$ as that seen in the type 1 diabetes offspring study [5], we would require a total of 18 and 62 subjects respectively.

\section{Results}

Of the 83 offspring, 46 were born to mothers with glucokinase mutations. There were no significant differ- 
ences in baseline characteristics between the two groups of subjects (Table 1). The mean birthweight (corrected for gestational age) in offspring born to mothers with glucokinase mutation was increased by $450 \mathrm{~g}$ compared with offspring of affected fathers $(p<0.001)$ (Fig. 1a). No significant differences in offspring beta cell function, as measured by EIR, 2-h glucose or insulin sensitivity, was seen in offspring exposed to maternal hyperglycaemia, compared with those not exposed (Fig. 1b-d). In cases compared with controls mean log EIR was 1.40 (95\%CI $1.28-1.52)$ vs $1.26(95 \% \mathrm{CI} 1.13-1.39), p=0.11$, and mean 2-h glucose was $9.1 \mathrm{mmol} / 1$ (95\%CI $8.1-10.0)$ vs $8.6 \mathrm{mmol} / 1$ (95\%CI 7.6-9.6), $p=0.50$. Adjusting for BMI did not alter these results.

When only the non-mutation-carrying offspring were considered, birthweight was increased by $720 \mathrm{~g}(p<0.001)$ in those born to mutation-carrying mothers (Fig. 1a), but again with no difference in any of the measures of glucose tolerance, beta cell dysfunction or other traits (Table 1, Fig. $1 b-d)$.

\section{Discussion}

We have shown that there is no difference in beta cell function or glucose tolerance between offspring born to mothers with heterozygous glucokinase mutations and offspring born to affected fathers. This is despite clear evidence of different intra-uterine exposure to hyperglycaemia as demonstrated by the marked increase in birthweight of offspring from mothers with glucokinase mutations. The glycaemia result is in keeping with a previous study of offspring from French glucokinase families, who showed no difference in glucose tolerance [10]. The size of our study and relative imprecision of our measurement of beta cell function means that we cannot exclude a small impact on beta cell function, but the

Table 1 Baseline offspring characteristics according to parent with glucokinase mutation

\begin{tabular}{|c|c|c|c|c|c|c|c|c|c|}
\hline \multirow[t]{2}{*}{ Offspring characteristics } & \multicolumn{3}{|c|}{ Non-mutation-carrying offspring } & \multicolumn{3}{|c|}{ Mutation-carrying offspring } & \multicolumn{3}{|c|}{$\begin{array}{l}\text { All offspring adjusted for mutation } \\
\text { status }\end{array}$} \\
\hline & $\begin{array}{l}\text { Mother } \\
\text { affected }\end{array}$ & $\begin{array}{l}\text { Father } \\
\text { affected }\end{array}$ & $\begin{array}{l}p \\
\text { value }\end{array}$ & $\begin{array}{l}\text { Mother } \\
\text { affected }\end{array}$ & $\begin{array}{l}\text { Father } \\
\text { affected }\end{array}$ & $\begin{array}{l}p \\
\text { value }\end{array}$ & $\begin{array}{l}\text { Mother } \\
\text { affected }\end{array}$ & $\begin{array}{l}\text { Father } \\
\text { affected }\end{array}$ & $\begin{array}{l}p \\
\text { value }\end{array}$ \\
\hline Number & 15 & 14 & & 31 & 23 & & 46 & 37 & \\
\hline Sex (male:female) & $4: 11$ & $7: 7$ & 0.20 & $9: 22$ & $6: 17$ & 0.81 & $13: 33$ & $13: 24$ & 0.50 \\
\hline Age (years) & $35.8(4.3)$ & $38.8(3.7)$ & 0.60 & $43.3(2.5)$ & $38.5(2.6)$ & 0.19 & $40.8(2.1)$ & $38.6(2.1)$ & 0.47 \\
\hline Proportion with IGT & $1 / 13$ & $1 / 14$ & 0.96 & $13 / 31$ & $12 / 23$ & 0.46 & $14 / 44$ & $13 / 37$ & 0.75 \\
\hline Proportion with diabetes & $0 / 13$ & $1 / 14$ & 0.33 & $13 / 31$ & $6 / 23$ & 0.23 & $13 / 44$ & $7 / 37$ & 0.27 \\
\hline BMI $\left(\mathrm{kg} / \mathrm{m}^{2}\right)^{\mathrm{a}}$ & $26.3(1.4)$ & $27.2(1.4)$ & 0.67 & $26.4(0.9)$ & $25.4(1.1)$ & 0.47 & $26.3(0.8)$ & $26.1(0.8)$ & 0.87 \\
\hline Waist $(\mathrm{m})^{\mathrm{a}}$ & $0.89(0.04)$ & $0.89(0.04)$ & 0.98 & $0.90(0.02)$ & $0.86(0.03)$ & 0.24 & $0.89(0.02)$ & $0.87(0.02)$ & 0.49 \\
\hline Body fat ${ }^{a}(\%)$ & $29.0(2.1)$ & $27.6(2.2)$ & 0.63 & $29.8(1.3)$ & $28.1(1.6)$ & 0.41 & $29.4(1.1)$ & $28.0(1.2)$ & 0.40 \\
\hline $\mathrm{HbA}_{1 \mathrm{c}}(\%)^{\mathrm{a}}$ & $5.34(0.07)$ & $5.27(0.07)$ & 0.56 & $6.54(0.17)$ & $6.37(0.19)$ & 0.51 & $6.16(0.11)$ & $5.98(0.12)$ & 0.30 \\
\hline Systolic $\mathrm{BP}^{\mathrm{a}}(\mathrm{mmHg})$ & $119(5)$ & $122(5)$ & 0.67 & $117(2)$ & $120(3)$ & 0.56 & $118(2)$ & $120(2)$ & 0.53 \\
\hline Diastolic $\mathrm{BP}^{\mathrm{a}}(\mathrm{mmHg})$ & $71(2)$ & $73(2)$ & 0.49 & $71(2)$ & $72(2)$ & 0.66 & $71(1)$ & $73(1)$ & 0.35 \\
\hline Fasting glucose $(\mathrm{mmol} / \mathrm{l})^{\mathrm{a}}$ & $4.98(0.09)$ & $5.01(0.10)$ & 0.83 & $7.39(0.35)$ & $7.19(0.41)$ & 0.71 & $6.60(0.24)$ & $6.37(0.26)$ & 0.51 \\
\hline $\begin{array}{l}\text { 30-min OGTT glucose } \\
(\mathrm{mmol} / \mathrm{l})^{\mathrm{a}}\end{array}$ & $7.58(0.38)$ & $7.65(0.38)$ & 0.90 & $11.16(0.46)$ & $10.18(0.53)$ & 0.18 & $10.04(0.33)$ & $9.22(0.37)$ & 0.10 \\
\hline $\begin{array}{l}\text { 60-min OGTT glucose } \\
(\mathrm{mmol} / \mathrm{l})^{\mathrm{a}}\end{array}$ & $7.63(0.51)$ & $6.51(0.51)$ & 0.14 & $11.59(0.73)$ & $10.59(0.63)$ & 0.31 & $10.36(0.46)$ & $9.05(0.50)$ & 0.06 \\
\hline $\begin{array}{l}\text { 90-min OGTT glucose } \\
(\mathrm{mmol} / \mathrm{l})^{\mathrm{a}}\end{array}$ & $6.16(0.44)$ & $5.88(0.44)$ & 0.66 & $11.22(0.69)$ & $10.49(0.80)$ & 0.50 & $9.62(0.49)$ & $8.77(0.54)$ & 0.24 \\
\hline $\begin{array}{l}\text { 120-min OGTT glucose } \\
(\mathrm{mmol} / 1)^{\mathrm{a}}\end{array}$ & $5.83(0.48)$ & $5.65(0.46)$ & 0.80 & $10.61(0.67)$ & $10.16(0.77)$ & 0.66 & $9.08(0.48)$ & $8.60(0.52)$ & 0.50 \\
\hline Fasting log insulin ${ }^{\mathrm{a}}$ & $1.99(0.05)$ & $1.98(0.05)$ & 0.97 & $1.94(0.03)$ & $1.86(0.04)$ & 0.12 & $1.96(0.03)$ & $1.90(0.03)$ & 0.20 \\
\hline 30-min OGTT log insulin ${ }^{\mathrm{a}}$ & $2.63(0.08)$ & $2.57(0.08)$ & 0.58 & $2.48(0.05)$ & $2.35(0.05)$ & 0.07 & $2.53(0.04)$ & $2.42(0.04)$ & 0.05 \\
\hline 60-min OGTT log insulin ${ }^{\mathrm{a}}$ & $2.64(0.09)$ & $2.58(0.09)$ & 0.67 & $2.50(0.05)$ & $2.34(0.06)$ & 0.05 & $2.55(0.04)$ & $2.42(0.05)$ & 0.05 \\
\hline 90-min OGTT log insulin ${ }^{\mathrm{a}}$ & $2.59(0.08)$ & $2.54(0.08)$ & 0.70 & $2.48(0.05)$ & $2.32(0.06)$ & 0.04 & $2.53(0.04)$ & $2.40(0.05)$ & 0.04 \\
\hline 120-min OGTT log insulin ${ }^{a}$ & $2.43(0.08)$ & $2.42(0.09)$ & 0.95 & $2.45(0.05)$ & $2.28(0.05)$ & 0.02 & $2.45(0.04)$ & $2.32(0.04)$ & 0.04 \\
\hline
\end{tabular}

Unless otherwise stated, data are shown as adjusted mean (SE). $p$ values were obtained by comparing means by $t$-test (age), chi-square (sex distribution, proportion with diabetes or IGT), or analysis of covariance using covariates age and sex. Means for all offspring were additionally corrected for glucokinase mutation carrier status.

Diabetes was defined as 2-h OGTT venous plasma $>11.1 \mathrm{mmol} / 1$; IGT was defined as 2-h OGTT venous plasma $>7.8 \mathrm{mmol} / 1 \mathrm{and} \leq 11.1 \mathrm{mmol} / 1$. $B P$ blood pressure 

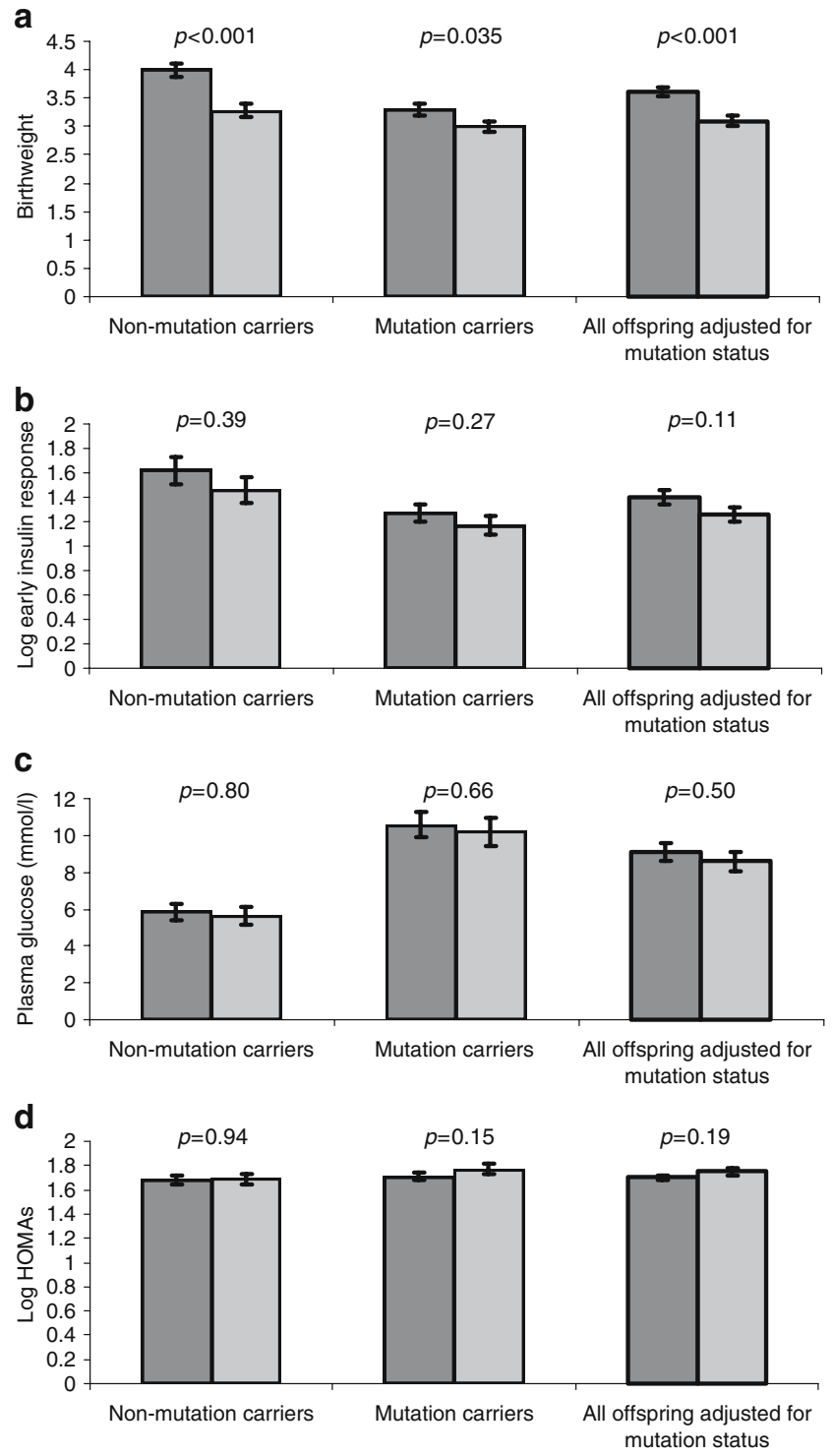

Fig. 1 Outcome in adult offspring by affection status of parent. a Offspring birthweight, $\mathbf{b}$ offspring early insulin response, $\mathbf{c}$ offspring 2-h glucose post OGTT, d offspring insulin sensitivity using homeostatic model assessment (HOMA). Dark bars, offspring born to mothers with glucokinase mutation; light bars, offspring born to fathers with glucokinase mutation. Vertical bars, standard error of mean. All mean values are adjusted for age and sex (except birthweight, which was corrected for gestational age and sex)

95\% confidence limits of our results also exclude the possibility of a reduction of beta cell function above $23 \%$ and a change in 2 -h glucose above $21 \%(1.8 \mathrm{mmol} / \mathrm{l})$.

Our results contrast with the clear impact of in utero exposure to maternal type 2 diabetes in Pima Indians [1, 2], and with results in subjects with hepatic nuclear factor lalpha mutations [3]. In both of these examples, the offspring probably experienced a similar degree of hyperglycaemia in utero to our offspring of glucokinase mothers. We propose that the difference in outcome reflects the possibility that these offspring were genetically predisposed to beta cell dysfunction, unlike the offspring in our study. In our previous study of white offspring born on average 20 years prior to parental type 2 diabetes, even very modest exposure to hyperglycaemia in utero reduced beta cell function in these genetically susceptible offspring [4].

Reduced beta cell function and worse glycaemia in offspring of type 1 diabetic mothers compared with offspring of affected fathers is the only example of an impact of hyperglycaemia exposure in utero in subjects who were not genetically predisposed to progressive beta cell dysfunction [5]. This result is interesting, as birthweight did not differ significantly between offspring who had maternal hyperglycaemia exposure in utero and those who did not, perhaps the result of excellent treatment of maternal hyperglycaemia during pregnancy. Our glucokinase offspring were older (mean age 40 vs 23 years) and had greater BMI (27 vs $23 \mathrm{~kg} / \mathrm{m}^{2}$ ) than the offspring born to parents with type 1 diabetes, but still did not show the increased prevalence of IGT seen in that study [5].

It is unclear why programming results should be seen in offspring of type 1 diabetic mothers, but not in offspring of glucokinase mothers, despite birthweight evidence suggesting greater maternal hyperglycaemia exposure and a larger sample size in the glucokinase subjects. It is possible that the larger glucose excursions seen in type 1 diabetes, perhaps in the first trimester, are more detrimental to fetal beta cell development than the more sustained but milder hyperglycaemic exposure in glucokinase subjects. Replication of the result in the offspring of type 1 diabetic parents in a larger cohort would support this possible explanation.

In conclusion, we did not show a reduction in beta cell function or deterioration in glucose tolerance in offspring exposed to moderate hyperglycaemia in utero as a result of maternal glucokinase mutations. As previous examples of marked programming in humans by hyperglycaemia in utero have been in genetically predisposed offspring, we propose that our finding reflects lack of genetic predisposition in the offspring to progressive beta cell dysfunction.

Acknowledgements This study was funded by Diabetes UK. We acknowledge the molecular genetic team at the Royal Devon \& Exeter NHS Trust, particularly A. Parrish for the molecular genetic analysis. We would also like to acknowledge all the patients and their referring clinicians.

Duality of interest The authors declare no duality of interest.

\section{References}

1. Pettitt D, Aleck K, Baird H, Carraher M, Bennett P, Knowler W (1988) Congenital susceptibility to NIDDM: role of intrauterine environment. Diabetes 37:622-628

2. Gautier J-F, Wilson C, Weyer C et al (2001) Low acute insulin secretory responses in adult offspring of people with early onset type 2 diabetes. Diabetes 50:1828-1833 
3. Stride A, Shepherd M, Frayling T, Bulman M, Ellard S, Hattersley A (2002) Intrauterine hyperglycemia is associated with an earlier diagnosis of diabetes in HNF-1a gene mutation carriers. Diabetes Care 25:2287-2291

4. Singh R, Pearson E, Avery PJ et al (2006) Reduced beta cell function in offspring of mothers with young-onset type 2 diabetes. Diabetologia 49:1876-1880

5. Sobngwi E, Boudou P, Mauvais-Jarvis F et al (2003) Effect of a diabetic environment in utero on predisposition to type 2 diabetes. Lancet 361:1861-1865

6. Stride A, Hattersley AT (2002) Different genes, different diabetes: lessons from maturity-onset diabetes of the young. Ann Med $34: 207-216$
7. Hattersley AT, Beards F, Ballantyne E, Appleton M, Harvey R, Ellard S (1998) Mutations in the glucokinase gene of the fetus result in reduced birth weight. Nat Genet 19:268-270

8. Wareham N, Phillips D, Byrne C, Hales C (1995) The 30 minute insulin incremental response in an oral glucose tolerance test as a measure of insulin secretion. Diabet Med 12:931

9. Levy JC, Matthews DR, Hermans MP (1998) Correct homeostasis model assessment (HOMA) evaluation uses the computer program. Diabetes Care 21:2191-2192

10. Velho G, Hattersley AT, Froguel P (2000) Maternal diabetes alters birth weight in glucokinase-deficient (MODY2) kindred but has no influence on adult weight, height, insulin secretion or insulin sensitivity. Diabetologia 43:1060-1063 